POS PROCEEDINGS

\title{
Scales and orders of magnitude in Astronomy
}

\author{
Javier Alcolea* \\ Observatorio Astronómico Nacional (Spain) \\ E-mail: j.alcolea@oan.es
}

As an introduction to the forthcoming units, I will review in this one the typical scales used in Astronomy for the most important physical magnitudes, such as size and distance, mass, time, speed, and energy.

2nd MCCT-SKADS Training School. Radio Astronomy: fundamentals and the new instruments August 26-September 4, 2008

Sigüenza, Spain

${ }^{*}$ Speaker. 


\section{Introduction: Astronomical units}

Astronomy is the science that studies the bodies, processes and phenomena occurring outside the Earth's atmosphere. Yet it also deals with substances that found in the Earth are of extraterrestrial origin. Because of its subjects of study, Astronomy is a very broad science, where observations have larger impact than laboratory studies. Astronomy deals with systems extremely large and at the same time very far away from us. These systems, may consist in much more smaller components, sometimes even of microscopic size. In addition, in Astronomy we study both very energetic processes as well as emissions arising from systems nearly at the absolute zero of temperature. These processes can be very fast, lasting for just a few seconds, or be very slow lasting thousands of million years.

All these characteristics make that the quantities measured in Astronomy may have a very large range of values, from very small figures to very large ones. The handling of very large or very small figures is not very practical, this is why in Astronomy we use a broad variety of units for the same physical magnitude. These units are typically used in those contexts for which they may result better suited. This general tendency in Astronomy difficults up to some point the comprehension of the relevant scales and orders of magnitude for those who are not very familiar with the field. Since this school is not intended just for radio astronomers, even not just for astronomers, as an introduction to the forthcoming units, I am going to review the typical scales used in Astronomy for the most important physical magnitudes: size and distance, mass, time, speed, and energy.

\section{Sizes and size units in Astronomy}

- Typical size nucleons

- Typical size of atoms and small molecules

- Circumstellar and interstellar dust grains

- Micro-meteorites

- Meteorites and comets

- Planets, satellites and large asteroids

- The Earth

- Mercury

- Jupiter

- The Moon

- Io

- Titan

- Ceres

- Stars

$$
\begin{array}{r}
\text { femtometers }\left(\mathrm{fm}=10^{-15} \mathrm{~m}\right) \\
\text { Ånströngs }\left(\AA=10^{-10} \mathrm{~m}\right) \\
\text { microns }\left(\mu \mathrm{m}=10^{-6} \mathrm{~m}\right) \\
\text { millimeters }\left(\mathrm{mm}=10^{-3} \mathrm{~m}\right) \\
\text { meters — kilometers }\left(\mathrm{m}, \mathrm{km}=10^{3} \mathrm{~m}\right) \\
\text { thousands of kilometers } \\
1.310^{4} \mathrm{~km} \\
4.910^{3} \mathrm{~km} \\
1.410^{5} \mathrm{~km} \\
3.510^{3} \mathrm{~km} \\
3.610^{3} \mathrm{~km} \\
5.110^{3} \mathrm{~km} ; \\
9.310^{2} \mathrm{~km}
\end{array}
$$

Solar radius $\left(\mathrm{R}_{\odot}=710^{5} \mathrm{~km}=4.610^{-3} \mathrm{AU}\right)$ 
- Dwarf stars

- Red giants and supergiants

- Planetary systems

- Orbit of Neptune

- Kuiper belt

- Molecular clouds

- Dark clouds

- Giant clouds

- Galaxies

- Spiral galaxies The Milky Way

- Elliptical galaxies

- Irregular galaxies
$0.01-0.1 \mathrm{R}_{\odot}$

$100-1000 \mathrm{R}_{\odot}$

Astronomical Units $\left(\mathrm{AU}=1.510^{8} \mathrm{~km}=4.810^{-6} \mathrm{pc}\right)$

$60 \mathrm{AU}$

$2000 \mathrm{AU}$

$\operatorname{parsecs}\left(\mathrm{pc}=310^{16} \mathrm{~m}=210^{5} \mathrm{AU}\right)$

$1-20 \mathrm{pc}$

$50-100 \mathrm{pc}$

kiloparsecs $\left(\mathrm{kpc}=10^{3} \mathrm{pc}=310^{19} \mathrm{~m}\right)$

$5-250 \mathrm{kpc}$ $50 \mathrm{kpc}$

$1-200 \mathrm{kpc}$

$1-10 \mathrm{kpc}$

- Active Galactic Nuclei (AGNs)

- Giant radio-emitting lobes in 3C273

- Central engine of the AGN (M 87)

- Galaxy clusters megaparsecs $\left(\mathrm{Mpc}=10^{+6} \mathrm{pc}=310^{22} \mathrm{~m}\right)$

- Cluster several Mpc

- Super-clusters and voids

\section{Distances in Astronomy}

- Planetary Systems

- Radius of the orbit of Mercury

- Radius of the orbit of the Earth

- Radius of the orbit of Neptune

- Radius of the orbit of the Moon

- Radius of the orbit of the innermost satellite of Jupiter

- Radius of the orbit of the outermost satellite of Jupiter

- Distances between stars in our Galaxy

- Distance from the Sun to Proxima Centauri

- Number of stars closer than $5 \mathrm{pc}$ to our Sun
Astronomical Units $\left(\mathrm{AU}=1.510^{8} \mathrm{~km}=4.810^{-6} \mathrm{pc}\right)$

$0.39 \mathrm{AU}$

$1 \mathrm{AU}$

$30 \mathrm{AU}$

$0.0025 \mathrm{AU}\left(3.810^{5} \mathrm{~km}\right)$

$0.0008 \mathrm{AU}\left(1.310^{5} \mathrm{~km}\right)$

$0.2 \mathrm{AU}\left(3.510^{7} \mathrm{~km}\right)$

several pc - several kpc

$1.3 \mathrm{pc}$

$\sim 40$ 
- Distance from the Sun to Taurus (dark molecular cloud)

$140 \mathrm{pc}$

- Distance from the Sun to Orion (giant molecular cloud complex)

$500 \mathrm{pc}$

- Distance from the Sun to the center of our Galaxy $8500 \mathrm{pc}(8.5 \mathrm{kpc})$

- Distance to other Galaxies in the Local Group

$$
\begin{array}{r}
\text { tens of } \mathrm{kpc}-\mathrm{Mpc} \\
50-60 \mathrm{kpc} \\
750 \mathrm{kpc} \\
2300 \mathrm{kpc}
\end{array}
$$

- Distance to the Magellanic Clouds

- Distance to Leo A (the farthest one)

- Distances to other nearby galaxies

up to $20-30 \mathrm{Mpc}$

- Distance to M51

$11 \mathrm{Mpc}$

- Distances to distant galaxies

hundreds to thousands of $\mathrm{Mpc}$

We can also use red-shift units $(z)$. Due to the Doppler effect, the wavelength $\lambda$ of the electromagnetic waves (EMWs) radiated by an emitter which is moving away from us a speed $v$, will change according to the law:

$$
\lambda=\lambda_{0}(1+z) \quad ; \quad z=\frac{\lambda-\lambda_{0}}{\lambda_{0}}=\sqrt{\frac{c+v}{c-v}}-1
$$

where $\lambda$ is the wavelength observed by us, $\lambda_{0}$ is the wavelength in the reference frame of the emitter (at rest) and $c$ is the speed of light in vacuum $\left(\sim 299792.458 \mathrm{~km} \mathrm{~s}^{-1}\right)$.

According to the Hubble law, the distance to the distant galaxies is proportional to the speed at which the galaxy is receding from us, as consequence of the Big Bang:

$$
D=\frac{v}{H_{0}} \quad ; \quad H_{0} \sim 75 \mathrm{~km} \mathrm{~s}^{-1} \mathrm{Mpc}^{-1} \quad ; \quad v=c \frac{(1+z)^{2}-1}{(1+z)^{2}+1}
$$

where $D$ is the distance in Mpc. (We are assuming that there has not been acceleration nor deceleration in the Universe since the Big Bang, i.e. $q_{0}=0$ and $\Lambda_{c}=0$, which today we know is not true). Therefore, knowing the red-shift we can estimate the distance to a very far away object. Using the parameters above we obtain the following conversion table:

$\begin{array}{lr}\text { - } & \mathrm{z}=0.1 \Rightarrow \mathrm{D}=380 \mathrm{Mpc} \\ \text { - } & \mathrm{z}=0.5 \Rightarrow \mathrm{D}=1500 \mathrm{Mpc} \\ \text { - } & \mathrm{z}=1.0 \Rightarrow \mathrm{D}=2400 \mathrm{Mpc} \\ \text { - Typical distance to quasars } & \mathrm{z}=3.0 \Rightarrow \mathrm{D}=3500 \mathrm{Mpc} \\ \text { - Dist. to the farthest radio-quasar } & \mathrm{z}=6.2 \Rightarrow \mathrm{D}=3850 \mathrm{Mpc} \\ \text { - Dist. to the most distant galaxy (with well known red-shift) } \mathrm{z}=6.96 \Rightarrow \mathrm{D}=3900 \mathrm{Mpc}\end{array}$




\section{Masses and mass units in Astronomy}

- Planets

- The Earth

- Planets

- Stars

- The Sun

- Normal stars

- Brown dwarfs

- Stellar size black holes mass of Jupiter $\left(\mathrm{M}_{\mathrm{Jup}}=1.910^{27} \mathrm{~kg} \approx 0.001 \mathrm{M}_{\odot}\right)$

$$
\begin{array}{r}
3.210^{-3} \mathrm{M}_{\text {Jup }} \\
<0.013 \mathrm{M}_{\odot}\left(<13 \mathrm{M}_{\text {Jup }}\right)
\end{array}
$$

solar masses $\left(\mathrm{M}_{\odot}=2.010^{30} \mathrm{~kg} \approx 1000 \mathrm{M}_{\text {Jup }}\right)$

$$
\begin{array}{r}
1 \mathrm{M}_{\odot} \\
0.08-100 \mathrm{M}_{\odot} \\
0.013-0.08 \mathrm{M}_{\odot} \\
\text { several } \mathrm{M}_{\odot}
\end{array}
$$

- Molecular clouds

- Taurus (dark molecular cloud)

$$
10^{4} \mathrm{M}_{\odot}
$$$$
510^{5} \mathrm{M}_{\odot}
$$

- Orion (giant molecular cloud)

- Nuclei of galaxies

- Super-massive black hole in Sgr A* (Milky Way)

$2.610^{6} \mathrm{M}_{\odot}$

- Super-massive black hole in a typical AGN

$$
10^{7}-10^{9} \mathrm{M}_{\odot}
$$

- Galaxies

- Spiral galaxies

$$
\begin{aligned}
& 10^{9}-410^{11} \mathrm{M}_{\odot} \\
& 10^{5}-10^{13} \mathrm{M}_{\odot} \\
& 10^{8}-310^{10} \mathrm{M}_{\odot}
\end{aligned}
$$

- Elliptical galaxies

\section{Energies and power units in Astronomy}

Rather than about Energy, in Astronomy we usually talk about Luminosity, which is the energy emitted by a certain source per unit of time, i.e. it is the power emitted by the object (not only by means of EMWs but by all means). Luminosities are always measured in units of the luminosity of the Sun, i.e. solar luminosities, $\mathrm{L}_{\odot}=3.910^{26} \mathrm{~W}\left(3.910^{33} \mathrm{erg} \mathrm{s}^{-1}\right)$.

- Stars

- Low mass stars and white dwarfs

$$
10^{-4}-10^{-2} \mathrm{~L}_{\odot}
$$

- Our Sun

$1 \mathrm{~L} \odot$

- Giant, super-giant, and hyper-giant stars

$10^{4}-10^{6} \mathrm{~L} \odot$

- Supernova explosions

$10^{8}-10^{10} \mathrm{~L} \odot$

- Galaxies 
- Normal spiral galaxies

- Normal elliptical galaxies

- Irregular galaxies

- Active galactic nuclei (AGNs)

$$
\begin{gathered}
10^{8}-210^{10} \mathrm{~L}_{\odot} \\
310^{5}-10^{11} \mathrm{~L}_{\odot} \\
10^{7}-10^{9} \mathrm{~L}_{\odot} \\
10^{8}-10^{14} \mathrm{~L}_{\odot}
\end{gathered}
$$

\section{Time scales in Astronomy}

Usually, time in Astronomy is measured as in normal life in seconds (s), minutes (m), hours (h), days (d) and years (yr). The most common unit is the year, since in general astronomical processes evolve very slowly in comparison with human standards. Nevertheless, for very fast events, such as explosive phenomena, variability, etc., seconds and days are the preferred units.

- Age of the Universe after WMAP results

$$
13.7 \mathrm{Gyr}\left(1.3710^{10} \mathrm{yr}=4.110^{17} \mathrm{~s}\right)
$$

- Age of the Universe at the end of the primordial nucleo-synthesis

- Age of the Universe at the Recombination Epoch $300.000 \mathrm{yr}$

- Age of the Sun and Solar System $4.6 \mathrm{Gyr}$

- Stars: duration of their lives while in the main sequence

- Star with $0.5 \mathrm{M}_{\odot}$ $200 \mathrm{Gyr}$

- Star with $1.0 \mathrm{M}_{\odot}$ $10 \mathrm{Gyr}$

- Star with 3.0 $\mathrm{M}_{\odot}$ $0.5 \mathrm{Gyr}$

- Star with $25 \mathrm{M}_{\odot}$ $0.005 \mathrm{Gyr}$

- Stellar variability seconds - years

- AGNs

- Duration of the activity period of AGNs $\sim$ several Gyr

- Variability timescale in AGN several hours - several tens of $\mathrm{yr}\left(10^{4}-10^{9} \mathrm{~s}\right)$

Sometimes, from the variability timescales we can roughly estimate the size of the emitting region along the line of sight. We just need to multiply the typical time scale of the fluctuations of the by the speed of light, to obtain the typical size of the emitting region.

\section{Speeds in Astronomy}

Speeds are normally measured in $\mathrm{km} \mathrm{s}^{-1}$, except when they are close to the speed of the light $c$, when they are usually given in units of fractions of $c$.

- Maximum possible speed, $c$ (just for massless particles) $299792.458 \mathrm{~km} \mathrm{~s}^{-1}$ 
- Receding speed of the most distant galaxy $(z=6.96)$

- Orbital velocities

$$
\frac{m v_{\mathrm{orb}}^{2}}{r}=G \frac{M m}{r^{2}} \Rightarrow v_{\mathrm{orb}}=\sqrt{\frac{M G}{r}}
$$

where $G$ is the Gravitational constant $\left(6.673(10) 10^{-11} \mathrm{~m}^{3} \mathrm{~kg}^{-1} \mathrm{~s}^{-2}\right)$ and $M$ is the mass of the attracting body.

- Orbital speed of the Moon (around the Earth)

$1 \mathrm{~km} \mathrm{~s}^{-1}$

- Orbital speed of the Earth (around the Sun)

- Orbital speed of Neptune (around the Sun)

- Orbital speed of the Sun (around the center of the Galaxy)

- Orbital speed of the S1 star (Sgr A*)

- Orbital speed of the central regions of M 84

- Orbital speed of the $\mathrm{H}_{2} \mathrm{O}$ masers in the Seyfert galaxy NGC 4258

- Ejection speeds. They are usually similar to the corresponding escape velocities $v_{\mathrm{esc}}$

$$
\frac{1}{2} m v_{\mathrm{esc}}^{2}=G \frac{M m}{r} \Rightarrow v_{\mathrm{esc}}=\sqrt{\frac{2 M G}{r}}
$$

- Escape velocity of the Earth

- Escape velocity of the Sun

- Expansion velocity in circumstellar envelopes of AGB stars

$10-20 \mathrm{~km} \mathrm{~s}^{-1}$

- Speed of the bipolar jets ejected by young/old stars up to several $100 \mathrm{~km} \mathrm{~s}^{-1}$

- Expansion speed of supernova remnants $\sim 1000 \mathrm{~km} \mathrm{~s}^{-1}$

- Speed of the bipolar jets ejected by AGNs some fraction of $c$

- Velocities due to thermal agitation in a gas

$$
\frac{1}{2} m v^{2}=\frac{1}{2} k T \quad \Rightarrow \quad v=\sqrt{\frac{k T}{m}} \equiv v\left(\mathrm{~km} \mathrm{~s}^{-1}\right)=0.09 \sqrt{\frac{T(\mathrm{~K})}{m(\mathrm{aum})}}
$$

where $T$ is the temperature of the gas, $k$ the Boltzman constant, and $m$ the mass of the gas particles.

- Velocities due to turbulent motions

some $\mathrm{km} \mathrm{s}^{-1}$ 
- Sound speed in a gas $v_{\mathrm{s}}$

$$
v_{\mathrm{s}}=\sqrt{\frac{\gamma k T}{m}}
$$

where $m$ is the average mass per gas particle. $\gamma$ takes $5 / 3$ or 1 depending on whether we talk about adiabatic sound speed or isothermal sound speed, respectively. Assuming an ideal gas consisting in molecular Hydrogen and atomic Helium in a 3 to 1 relationship, we have:

$$
m=2.25(\text { uma }) \quad ; \quad v_{\mathrm{s}}\left(\mathrm{km} \mathrm{s}^{-1}\right)=0.07 \sqrt{\gamma T(\mathrm{~K})}
$$

\section{References}

[1] C.W. Allen, Astrophysical Quantities $\left(3^{\text {rd }}\right)$ edition, University of London, Athlone Press.

[2] Observatorio Astronómico Nacional, Anuario del Observatorio Astronómico de Madrid para 2008, Instituto Geográfico Nacional, Gral. Ibáñez de Ibero 3, Madrid (Spain).

[3] U.S. Naval Observatory and Her Majesty's Nautical Almanac Office, The Astronomical Almanac for the Year 2008, The U.S. Government Printing Office, 710 North Capitol Street N.W., Washington DC, USA. 